\title{
Accuracy of QuantiFERON-TB Gold Test for Tuberculosis Diagnosis in Children
}

\author{
Michela Sali ${ }^{1}$, Danilo Buonsenso ${ }^{2}$, Delia Goletti ${ }^{3}$, Pamela D'Alfonso ${ }^{1}$, Antonella Zumbo ${ }^{1}$, \\ Giovanni Fadda ${ }^{1}$, Maurizio Sanguinetti ${ }^{1}$, Giovanni Delogu' ${ }^{1}$, Piero Valentini' ${ }^{2 *}$ \\ 1 Institute of Microbiology, Università Cattolica del Sacro Cuore, Rome, Italy, 2 Institute of Pediatrics, \\ Università Cattolica del Sacro Cuore, Rome, Italy, 3 Translational Research Unit, Department of \\ Epidemiology and Preclinical Research, "Lazzaro Spallanzani" National Institute for Infectious Diseases \\ (INMI), IRCCS, Rome, Italy \\ * pvalentini@ rm.unicatt.it
}

\section{Abstract}

\section{Objectives}

To evaluate the accuracy of the QuantiFERON-TB Gold assay (QFT-IT) in children with suspected active or latent TB infection (LTBI).

\section{G openaccess}

Citation: Sali M, Buonsenso D, Goletti D, D'Alfonso P, Zumbo A, Fadda G, et al. (2015) Accuracy of QuantiFERON-TB Gold Test for Tuberculosis Diagnosis in Children. PLoS ONE 10(10): e0138952. doi:10.1371/journal.pone.0138952

Editor: Lei Gao, Chinese Academy of Medical Sciences and Peking Union Medical College, CHINA

Received: June 17, 2015

Accepted: September 5, 2015

Published: October 6, 2015

Copyright: @ 2015 Sali et al. This is an open access article distributed under the terms of the Creative Commons Attribution License, which permits unrestricted use, distribution, and reproduction in any medium, provided the original author and source are credited.

Data Availability Statement: All relevant data are within the paper.

Funding: The authors received no specific funding for this work.

Competing Interests: The authors have declared that no competing interests exist.

\section{Methods}

A retrospective study was conducted on 621 children (0-14 years old) evaluated for TB infection or disease. Following clinical assessment, children were tested with the QFT-IT assay.

\section{Results}

Among the 140 active TB suspects, we identified 19 cases of active disease. The overall sensitivity for active TB was $87.5 \%$, ranging from $62.5 \%$ in children $25-36$ months old to $100 \%$ in children older than 49 months. The overall specificity for active TB was $93.6 \%$. Among the 481 children tested for LTBI screening, 38 scored positive and all but 2 had at least one risk factor for TB infection. Among the 26 children with indeterminate results, bacterial, viral or fungal pneumonia were later diagnosed in 11 (42.3\%) cases and non-TB related extra-pulmonary infections in 12 (46.1\%).

\section{Conclusions}

Our results indicate that the children's response to QFT-IT associates to active TB and risk factors for $\mathrm{LTBI}$. Moreover, we show that mitogen response is also found in children of 1 year of age, providing support for QFT-IT use also in young children. 


\section{Introduction}

Children bear a substantial part of the tuberculosis (TB) epidemic at a global level. It is estimated that there were 530,000 childhood TB cases globally in 2013 [1], although it is problematic to obtain accurate data due to the many difficulties associated with TB diagnosis in children and the weakness of surveillance systems in countries where TB is endemic [2]. In children, the main risk factor for infection is exposure to adults with pulmonary TB, which, in children under the age of 2 , is very likely to progress to active disease within the first year of primary infection [3]. Moreover, young children are more likely to develop the most severe forms of disseminated and meningeal TB, characterized by high mortality rate [4]. For these reasons, children under the age of 5 with no signs of TB disease but who have close contact with a contagious adult TB patient should receive isoniazid preventive therapy (IPT), which is known to drastically reduce the risk of developing active TB [4]. Ideally, IPT should only be administered to truly infected children, but the lack of effective diagnostic tools prevents this approach.

Diagnosis of childhood TB is notoriously challenging due to non-specific clinical and radiological signs and difficulty in obtaining a microbiological confirmation. TB disease is often diagnosed by a positive Tuberculin Skin Test (TST) result, epidemiologic information (exposure to a known source case) and a compatible clinical and radiographic presentation. For decades, the TST was the only test available for diagnosing latent TB infection (LTBI), though both false-negative and false-positive results plague this old assay [4].

More recently, interferon- release assays (IGRAs) have been developed to replace the TST for detecting LTBI, and while their intended use is not to diagnose active TB, some studies suggested their usefulness to identify childhood TB [5]. The sensitivity and specificity of the TST and IGRAs were shown to be similar for diagnosing active TB, while it remains difficult to determine their accuracy in detecting LTBI, given the lack of gold standards in children and adults [6] and the somehow controversial definition of LTBI [7,8]. In this regard, the Paediatric Tuberculosis Network European Trials group (PTBNET) has recently defined LTBI as 'a positive immunological test result (either TST or IGRA) in the absence of active TB' [9]. As stated by the PTBNET, the proposed definition has a number of limitation, particularly in light of recent advances in TB pathogenesis. In fact, there is a growing understanding that the simple dichotomous classification of TB as "active" or "latent" is no longer acceptable and "TB infection" encompasses a wide spectrum of conditions, ranging from asymptomatic to lethal disease [10]. The lack of a clear edge between infection and disease is a major challenge for the clinician who face the problem to translate this new scenario in the routine clinical practice.

Although the TST has been widely used in clinical practice, it has known limitations, such as variable specificity, reproducibility, and cross-reactivity with non-tuberculous mycobacteria and BCG in those who have been vaccinated [11]. A major benefit of QuantiFERON-TB GoldIn-Tube (QFT-IT) (as well as other IGRAs) rests on its higher specificity in BCG-vaccinated subjects [12], preventing unnecessary and potentially toxic treatments [13-15]. However, the reliability of IGRAs in pediatric patients has been questioned. This is due to the lack of evidence of its performance in children under 5 years of age [16-18], (particularly in those under 2 years of age) mainly because of the reported high rate of indeterminate results and high variability among different studies. Indeed, even the recent NICE (National Institute for Health and Care Excellence) guidelines recommend the use of the TST rather than IGRAs for TB diagnosis in young children $[19,20]$.

In this 4 -year retrospective cross-sectional study, we aimed to evaluate the accuracy of the QFT-IT among young children of different ages who were evaluated for LTBI screening and enrolled with suspected active TB. 


\section{Methods}

\section{Study Setting and Population}

A retrospective cross-sectional study was conducted among children aged 0 to 14 years who were evaluated for TB infection or TB disease and referred to the Pediatric Infectious Disease Unit or outpatient clinic for the evaluation of internationally adopted children at the Catholic University of the Sacred Heart-A. Gemelli Hospital in Rome, Italy, during the period from January 2007 through July 2010.

The following category of children were enrolled in the study:

- Children with suspected active TB (symptomatic patients-children with suspected active TB)

- Children with known exposure to an active TB adult case (therefore children screened for LTBI);

- Clinically healthy, nationally or internationally adopted children evaluated by a national protocol for immigrants and nationally/internationally adopted children (see reference 12 for detailed list of screening tests performed), with or without known history of contact with adult active TB cases (therefore children screened for LTBI) approved by the National Working Group for Immigrant and Adopted Children, an official workinggroup of the Italian Society of Pediatrics and approved by the review board of our University.

Demographic and socio-economic characteristics, as well as complete personal and family histories in relation to TB exposure were recorded.

All patients were clinically evaluated and tested by QFT-IT (Cellestis Limited, Carnegie, Victoria, Australia). All children with a positive QFT-IT or high clinical suspicion of TB disease underwent radiological and microbiological investigations to confirm or rule out a diagnosis of active TB. Microbiological diagnosis included an acid-fast bacilli (AFB) examination following Ziehl-Neelsen staining; culture for Mycobacterium tuberculosis on Lowenstein-Jensen medium and in liquid media (BACTEC MGIT 960, Beckton-Dickinson, Maryland, USA); Strand Displacement Amplification (SDA) with PROBETEC ET ${ }^{\circledR}$ (Beckton-Dickinson, Maryland, USA) for M. tuberculosis. Gastric washings were obtained on 3 consecutive days from all patients with suspected active TB. From those with suspected extra-pulmonary TB, samples were taken from different sites according to the suspected TB localization.

All children with a final diagnosis of active TB were evaluated for HIV infection using the combined serologic test Abbott Architect HIV COMBO-BioMérieux Vidas HIV DUO.

Diagnosis of LTBI was performed based on positive QFT-IT and normal chest radiography and in the absence of any clinical features that would suggest active disease [21]. Children who were diagnosed with LTBI were offered chemoprophylaxis with 6 months of isoniazid, in accordance with NICE guidelines [19].

Two definitions of active TB were accepted: definite (confirmed) and probable TB.

Definite TB was defined as the presence of at least one clinical specimen positive for $M$. tuberculosis on culture, positive acid-fast bacilli smear microscopy, or one histology sample positive for caseating granulomas or nucleic acid amplification test. Probable TB was defined as the presence of three or more of the following: 1) chest radiologic findings consistent with active TB; 2) typical symptoms such as fever, cough and weight loss; 3 ) other radiological evidence of active TB, including extra-pulmonary TB (e.g., computed tomography/magnetic resonance imaging findings consistent with TB meningitis) in addition to symptoms; 4) exposure to a case with active infectious TB; and 5) response to appropriate anti-TB therapy. We then 
classified sites of TB as pulmonary and extra-pulmonary TB. Children with active TB were treated according to the current NICE international guidelines [19].

Therefore, according to the definitions used, the following final diagnoses were performed:

- active TB

- LTBI

- Non TB children (children with no active TB neither LTBI); this group include both healthy/asymptomatic children without LTBI (Healthy) and all children with any diagnosis other than TB, bacterial, viral, fungal or parasitic infection (disease other than TB$\operatorname{dot} \mathrm{TB})$

Conventionally, we will define "foreign children" all children born out of Italy and children born in Italy by non-Italian parents. This is an internationally accepted classification since it has been clearly demonstrated that children born in TB endemic countries have a similar risk of contracting TB of children born in Italy from parents born in TB endemic countries, and both have a higher risk than children born in Italy from Italian parents [5].

\section{QFT-IT Assay}

QFT-IT was performed as indicated by the manufacturer. Briefly, whole blood was collected in the QFT-IT tubes (Nil Control, TB-Ag and Mitogen) and incubated at $37^{\circ} \mathrm{C}$ for $16-24$ hours. Following incubation, samples were centrifuged and the plasma was used to measure the IFN- $\gamma$ produced in response to $M$. tuberculosis antigens, phytohaemagglutinin (PHA) and the negative control. Data were presented as IU/ml of IFN- $\gamma$; the cut-off value for a positive test was $0.35 \mathrm{IU} / \mathrm{ml}$, according to manufacturer's instructions.

\section{Statistical Analyses}

Differences in frequencies were evaluated by the Fisher Exact Test. The median and IQR of IFN- $\gamma$ production were calculated; the non-parametric Mann-Whitney U test was used to compare medians for unpaired comparisons and the Wilcoxon test for paired comparisons; the Kruskal-Wallis test was used to compare medians among the different groups. Data were analyzed using SPSS (SPSS, Chicago, IL) and Prism 5 software (Graphpad Software 5.0, San Diego, CA, USA). Differences were considered significant at $\mathrm{p}$ values $\leq 0.05$.

\section{Ethical statement}

This study was approved by the institutional review board of the Catholic University of Sacred Heart of Rome, Italy (prot 06/2015). The authors obtained verbal and written informed consent from the next of kin, caretakers, or guardians on behalf of the minors/children enrolled in the study.

\section{Results}

\section{Study population}

We evaluated 621 children, 275 (44.3\%) were female; Fig 1 summarizes the children evaluated as patients with suspected active TB and children screened for LTBI. The mean age of the study population was 49 months (range 0 to 140 months) (Table 1). Regarding the origins, 180 children $(28.9 \%)$ were Italian and $441(71.0 \%)$ were foreigners, mostly born outside of Italy (394; $89.3 \%)$, and only $47(10.6 \%)$ were born to foreign parents in Italy. All of the foreign children came from middle or high TB-burden countries (East Europe, Africa, South America, Asia) 


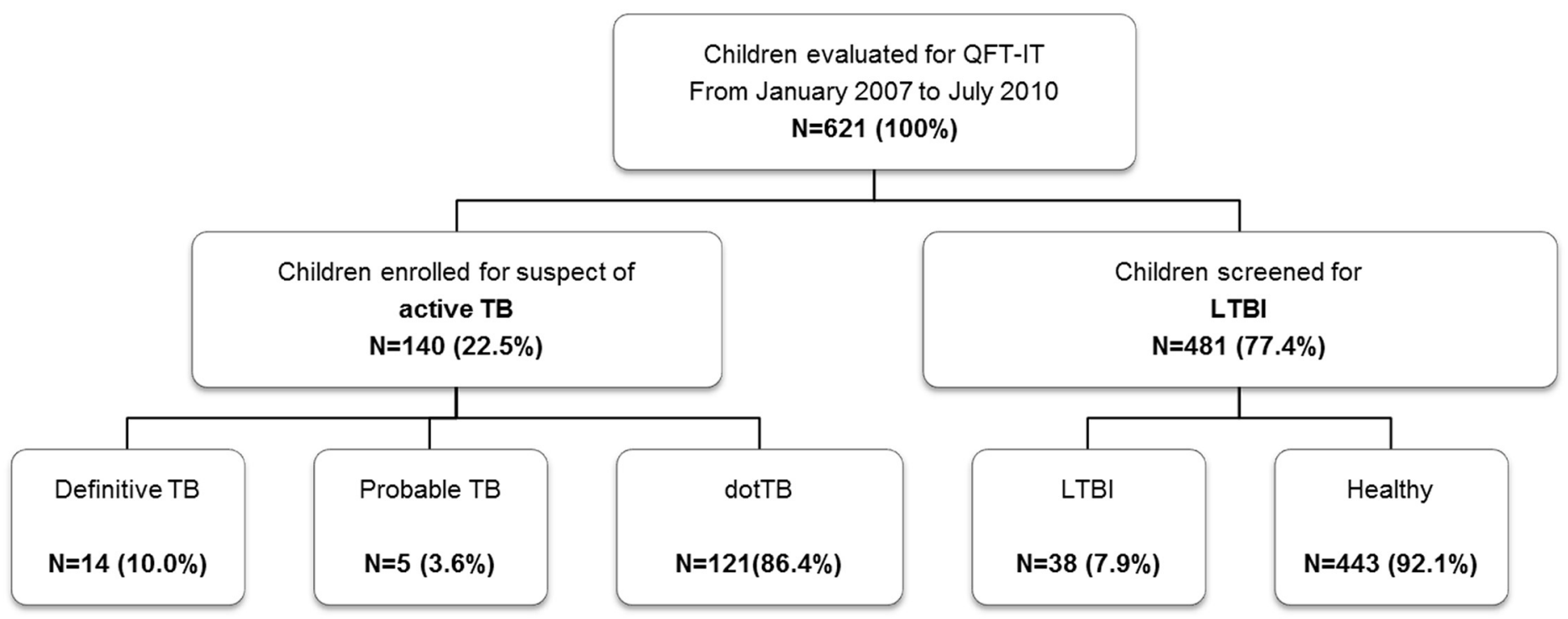

Fig 1. Flow chart of the study. (Abbreviations: TB: tuberculosis; LTBI: latent TB infection; QFT-IT: QUANTIferon TB Gold in tube.)

doi:10.1371/journal.pone.0138952.g001

and most of them were internationally adopted children $(352,56.5 \%$ of the total study population).

Overall, of the 621 children tested by the QFT-IT assay 59 (9.5\%) scored positive, 536 (86.3\%) scored negative and 26 (4.2\%) were given an indeterminate result (Table 2). Among the 19 children with active TB, 17 resulted positive, 2 had an indeterminate result (with a lack of response to nil and mitogen) and none scored negative, providing a specificity of $100 \%$ for the QFT-IT.

\section{Children evaluated for active TB}

Among the 140 children with suspected active TB, we identified 12 cases (57\%) of pulmonary TB, 4 cases (19\%) of extra-pulmonary TB ( 2 central nervous system, CNS TB, 2 lymph nodal TB); 5 cases (24\%) of pulmonary and extra-pulmonary TB ( 4 cases of pulmonary and CNS TB,

Table 1. Demographic characteristics of the study population.

\begin{tabular}{|c|c|c|c|c|c|c|c|c|}
\hline & & \multirow{3}{*}{$\frac{\frac{\text { Total N (\%) }}{621(100)}}{\text { Total N (\%) }}$} & \multicolumn{3}{|c|}{ Suspect of active TB N (\%) } & \multirow{2}{*}{\multicolumn{2}{|c|}{$\begin{array}{c}\text { LTBI screening N (\%) } \\
481(77.4)\end{array}$}} & \multirow{5}{*}{$P$ value } \\
\hline & & & \multicolumn{3}{|c|}{$140(22.5)$} & & & \\
\hline & & & \multicolumn{2}{|c|}{ active TB N (\%) } & \multirow[t]{2}{*}{ dotTB N (\%) } & \multirow[t]{2}{*}{ LTBI N (\%) } & \multirow[t]{2}{*}{ Non TB N (\%) } & \\
\hline & & & Definitive & Probable & & & & \\
\hline & & $621(100)$ & $14(10.0)$ & $5(3.6)$ & $121(86.4)$ & $38(7.9)$ & $443(92.1)$ & \\
\hline Sex & Female & $275(44.3)$ & $7(50)$ & $1(20.0)$ & $54(44.6)$ & $17(44.7)$ & $196(44.2)$ & 0.844 \\
\hline Age (months) & Median (IQR) & $49(22-79)$ & $16.5(11.7-31.5)$ & $29(14-32.5)$ & $40(19-76)$ & $67(39.5-91.5)$ & $51(23-79)$ & 0.001 \\
\hline \multirow[t]{2}{*}{ Classes of age } & 0-60 months & $378(60.9)$ & $13(92.9)$ & $5(100)$ & $85(70.2)$ & $16(42.1)$ & $259(58.4)$ & 0.0001 \\
\hline & $>60$ months & $243(39.1)$ & $1(7.1)$ & 0 & $36(29.7)$ & $22(59.4)$ & $184(41.4)$ & \\
\hline \multirow[t]{2}{*}{ Origin } & Italians & $180(29)$ & $2(14.2)$ & $1(20.0)$ & $77(63.6)$ & $7(18.4)$ & $95(21.4)$ & 0.0001 \\
\hline & Foreign children & $441(71)$ & $12(85.7)$ & $4(80.0)$ & $44(36.4)$ & $31(83.4)$ & $348(78.6)$ & \\
\hline
\end{tabular}

Footnotes: TB: tuberculosis; LTBI: latent tuberculosis infection; dotTb: disease other than TB

doi:10.1371/journal.pone.0138952.t001 
Table 2. QuantiFERON TB Gold in tube (QFT-IT) results as a function of the diagnosis.

\begin{tabular}{|c|c|c|c|c|c|c|c|c|}
\hline & \multirow{4}{*}{$\frac{\text { Total N (\%) }}{621(100)}$} & \multicolumn{3}{|c|}{ Suspect of active TB N (\%) } & \multirow{4}{*}{$p$ value } & \multirow{2}{*}{\multicolumn{2}{|c|}{$\begin{array}{c}\text { LTBI screening } N(\%) \\
481(77.4)\end{array}$}} & \multirow{4}{*}{ p value } \\
\hline & & \multicolumn{3}{|c|}{$140(22.5)$} & & & & \\
\hline & & \multicolumn{2}{|c|}{ active TB $N(\%)$} & \multirow[t]{2}{*}{$\operatorname{dotTB} N(\%)$} & & \multirow[t]{2}{*}{ LTBI N (\%) } & \multirow[t]{2}{*}{ Non TB N (\%) } & \\
\hline & & Definitive & Probable & & & & & \\
\hline & $621(100)$ & $14(10.0)$ & $5(3.6)$ & $121(86.4)$ & 0.0001 & $38(7.9)^{*}$ & $443(92.1)$ & $\mathrm{N} / \mathrm{A}$ \\
\hline QTF responders N (\%) & $59(9.5)$ & $13(92.9)$ & $4(80.0)$ & $4(3.3)$ & & $38(100)$ & 0 & \\
\hline QTF non responders $\mathrm{N}(\%)$ & $536(86.3)$ & 0 & 0 & 96 (79.3) & & 0 & $440(99.1)$ & \\
\hline QTF indeterminate N (\%) & $26(4.2)$ & $1(7.1)$ & $1(20.0)$ & $21(17.4)$ & & 0 & $3(0.7)$ & \\
\hline
\end{tabular}

Footnotes: TB: tuberculosis; LTBI: latent tuberculosis infection; dotTb: disease other than TB

* by definition

doi:10.1371/journal.pone.0138952.t002

1 case of pulmonary and chest wall TB). Microbiological confirmation was demonstrated in 14 (74\%) children, while an alternative final diagnosis (dotTB) was made for 121.

\section{Children evaluated for LTBI}

Among the 481 children tested for LTBI screening, 38 scored positive and all but 2 had at least one risk factor for $\mathrm{TB}$ infection (either known exposure with an adult index case, having been born in a TB-endemic country or having parents who were born in a TB-endemic country).

\section{Response to QFT-IT and age}

To investigate the relationship between age and ability to respond to the TB-specific antigens used in QFT-IT, we analyzed the IFN- $\gamma$ response of all children showing a positive result (Table 3, Fig 2A). The proportion of positivity to QFT-IT ranged from $7.8 \%$ for the $25-36$ months of age group to $10.9 \%$ for the 37-48 months of age group (Table 3), although the real significance of this value remains questionable given the non-homogeneity among the different age groups. Only 4 out of 52 children aged $\leq 12$ months scored positive by QFT-IT; three of these children were diagnosed as active TB, while the fourth was a healthy child born in a TBendemic country, diagnosed with LTBI. To note: one eight-month-old child with severe meningeal and cerebral TB (who did not survive) was scored QFT-IT indeterminate. In the 13-24 months age group, 11 out of 117 children scored positive and a final diagnosis of active TB was made for 7 of them. The remaining 4 children were diagnosed as LTBI: one child was a family contact of a smear-positive active TB adult, two children were born in TB-endemic countries, and for one child it was not possible to identify the risk factor for TB infection. In the 25-36 months of age group, six children scored QFT-IT positive and 5 of them were diagnosed as active TB. In the other three age groups ( $>36$ months), of the 38 children who scored positive by the QFT-IT, only two were diagnosed with active TB. Taken together, these results indicate that among the 21 QFT-IT -positive children $\leq 36$ months, 15 were active TB and the remaining six were diagnosed as LTBI with a good correlation with TB risk factors.

As shown in Fig 2A, all children with a positive QFT-IT test had an IFN- $\gamma$ production in response to TB-specific antigens which was always $>0.53 \mathrm{IU} / \mathrm{ml}$, while most of the negative results had IFN- $\gamma$ values $<0.2 \mathrm{IU} / \mathrm{ml}$. 
Table 3. QuantiFERON TB Gold in tube (QFT-IT) results as a function of the age.

\begin{tabular}{|c|c|c|c|c|c|c|c|}
\hline \multirow[b]{5}{*}{ age classes } & & \multirow{5}{*}{$\begin{array}{l}\frac{\text { Total N }}{621(100)} \\
621(100)\end{array}$} & \multirow{2}{*}{\multicolumn{3}{|c|}{$\begin{array}{c}\text { Suspect of active TB N (\%) } \\
140(22.5)\end{array}$}} & \multirow{2}{*}{\multicolumn{2}{|c|}{$\begin{array}{c}\text { LTBI screening } N(\%) \\
481(77.4)\end{array}$}} \\
\hline & & & & & & & \\
\hline & & & \multicolumn{2}{|c|}{ active TB N (\%) } & \multirow{3}{*}{$\begin{array}{c}\text { dotTB N (\%) } \\
121(86.4)\end{array}$} & \multirow{3}{*}{$\begin{array}{l}\underline{\text { LTBI N (\%) }} \\
38(7.9)\end{array}$} & \multirow{3}{*}{$\begin{array}{l}\text { Healthy N (\% } \\
443(92.1)\end{array}$} \\
\hline & & & Definitive & Probable & & & \\
\hline & & & $14(10.0)$ & $5(3.6)$ & & & \\
\hline \multirow[t]{3}{*}{$0-12$} & negative & $39(75.0)$ & 0 & 0 & $14(11.6)$ & 0 & $25(5.6)$ \\
\hline & positive & $4(7.7)$ & $2(14.2)$ & $1(20.0)$ & 0 & $1(2.7)$ & 0 \\
\hline & indeterminate & $9(13.7)$ & $1(7.1)$ & 0 & $7(5.8)$ & 0 & $1(0.2)$ \\
\hline \multirow[t]{3}{*}{$13-24$} & negative & $103(88.0)$ & 0 & 0 & $14(11.6)$ & 0 & $89(20.0)$ \\
\hline & positive & $11(9.4)$ & $6(42.9)$ & $1(20.0)$ & 0 & $4(10.5)$ & 0 \\
\hline & indeterminate & $3(2.6)$ & 0 & 0 & $3(2.5)$ & 0 & 0 \\
\hline \multirow[t]{3}{*}{$25-36$} & negative & $67(87.0)$ & 0 & 0 & $17(14.0)$ & 0 & $50(11.3)$ \\
\hline & positive & $6(7.8)$ & $3(21.4)$ & $2(40.0)$ & 0 & $1(2.7)$ & 0 \\
\hline & indeterminate & $4(5.2)$ & 0 & $1(20.0)$ & $3(2.5)$ & 0 & 0 \\
\hline \multirow[t]{3}{*}{$37-48$} & negative & $53(82.8)$ & - & - & $8(6.7)$ & 0 & $45(10.7)$ \\
\hline & positive & 7 (10.9) & - & - & $1(0.8)$ & $6(16.2)$ & 0 \\
\hline & indeterminate & $4(6.3)$ & - & - & $3(2.5)$ & 0 & $1(0.2)$ \\
\hline \multirow[t]{3}{*}{$49-60$} & negative & $59(86.8)$ & 0 & - & $11(9.1)$ & 0 & $48(10.8)$ \\
\hline & positive & $6(8.8)$ & $1(7.1)$ & - & $1(0.8)$ & $4(10.8)$ & 0 \\
\hline & indeterminate & $3(4.4)$ & 0 & - & $3(2.5)$ & 0 & 0 \\
\hline \multirow[t]{3}{*}{$>60$} & negative & 215 (88.5) & 0 & - & $32(26.4)$ & 0 & $183(41.2)$ \\
\hline & positive & 25 (10.3) & $1(7.1)$ & - & $2(1.6)$ & $22(5.9)$ & 0 \\
\hline & indeterminate & $3(1.2)$ & 0 & - & $2(1.6)$ & 0 & $1(0.2)$ \\
\hline
\end{tabular}

Footnotes: TB: tuberculosis; LTBI: latent tuberculosis infection; dotTb: Disease other than TB.

doi:10.1371/journal.pone.0138952.t003

(A)

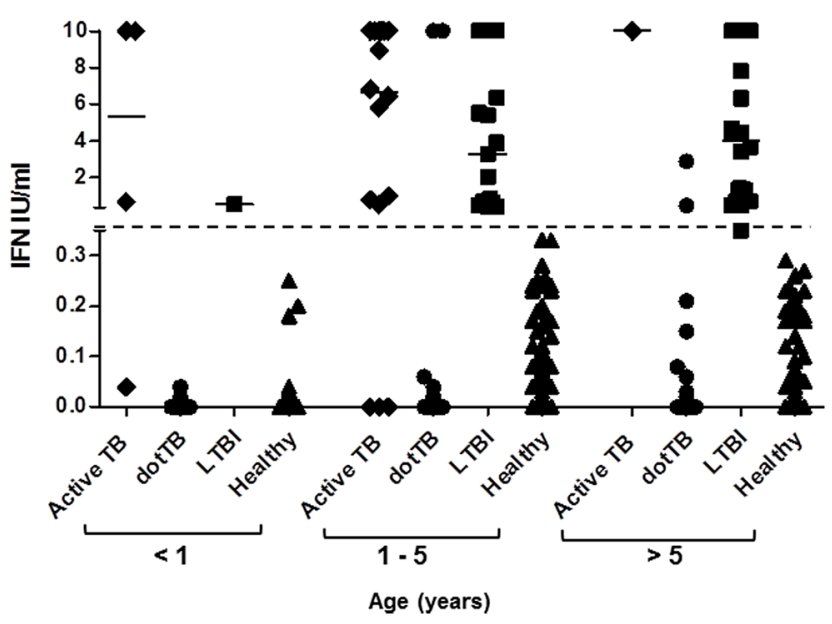

(B)

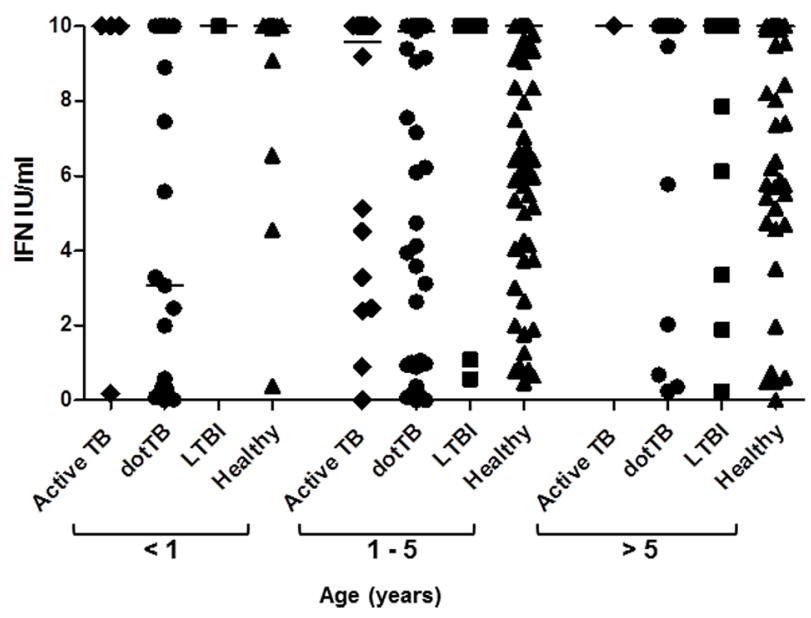

Fig 2. Quantitative response to QuantiFeron TB Gold-In Tube in relationship to age. IFN- $y$ levels in response to stimulation with $M$. tuberculosis antigens (A) and mitogen (B); individual QFT-IT results are plotted according to their final diagnosis and age. The cut-off value for a positive QFT-IT is represented by the dotted line at $0.35 \mathrm{IU} / \mathrm{ml}$.

doi:10.1371/journal.pone.0138952.g002 
Table 4. Clinical characteristics of the 121 patients enrolled for active TB but with an alternative diagnosis (dotTB).

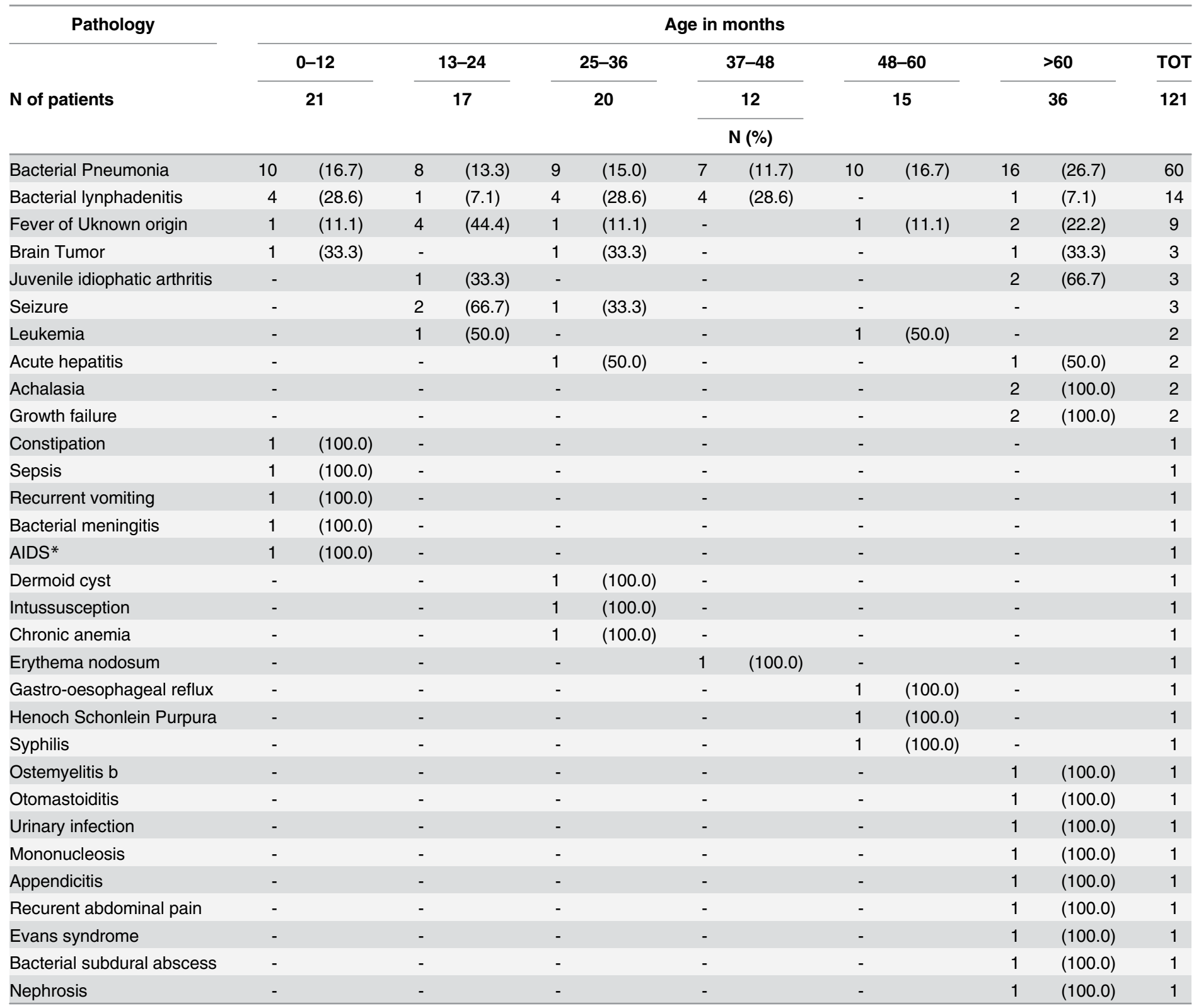

Footnote: AIDS: acquired immunedeficiency syndrome.

doi:10.1371/journal.pone.0138952.t004

\section{Mitogen and indeterminate results}

It has been suggested that the immune system in newborns and infants may not be properly mature or capable of responding to antigenic stimuli ex vivo and for these reasons caution has been recommended for the use of the IGRAs in this population [22]. Measurement of the IFN$\gamma$ secretion in response to mitogen in IGRAs provides a useful indication of the potential ability of the immune system to respond to the antigenic stimuli. As shown in Fig 2B and Table 4, most of the children in the different age groups responded to the mitogen by secreting high levels of IFN- $\gamma$, indicating that the immune system is not impaired in its ability to mount an 
Table 5. Clinical characteristics of the children scored indeterminate to QFT-IT.

\begin{tabular}{|c|c|c|}
\hline Age (months) & Diagnosis & Group \\
\hline 0 & Healthy newborn ( $6^{\text {th }}$ day of life) & Healthy \\
\hline 4 & Intracerebral space-occupying process & $\operatorname{dotTB}$ \\
\hline 6 & Pneumonia and Ongoing measles infection & $\operatorname{dotTB}$ \\
\hline 7 & CMV pneumonia & $\operatorname{dot} T B$ \\
\hline 7 & Acute respiratory failure in Congenital HIV infection & $\operatorname{dot} \mathrm{TB}$ \\
\hline 8 & Severe meningeal and cerebral TB-The child died because of CNS TB & active TB \\
\hline 8 & Pneumonia & $\operatorname{dotTB}$ \\
\hline 8 & Pneumonia & $\operatorname{dot} T B$ \\
\hline 10 & Bacterial lymphadenitis & $\operatorname{dotTB}$ \\
\hline 14 & Juvenile idiopathic arthritis & $\operatorname{dotTB}$ \\
\hline 16 & Acute myeloid leukemia & $\operatorname{dotTB}$ \\
\hline 20 & Fever of unknown origin & $\operatorname{dot} T B$ \\
\hline 29 & Bacterial lymphadenitis in ongoing chickenpox & $\operatorname{dotTB}$ \\
\hline 32 & Pulmonary TB (probable) & active TB \\
\hline 34 & Candida pneumonia in ongoing Epstein Barr infection & $\operatorname{dotTB}$ \\
\hline 36 & Pneumonia & $\operatorname{dotTB}$ \\
\hline 40 & Respiratory distress & $\operatorname{dotTB}$ \\
\hline 42 & Bacterial lymphadenitis & $\operatorname{dotTB}$ \\
\hline 42 & Healthy child & Healthy \\
\hline 45 & Bacterial lymphadenitis & $\operatorname{dotTB}$ \\
\hline 50 & Pneumonia & $\operatorname{dotTB}$ \\
\hline 50 & Pneumonia & $\operatorname{dotTB}$ \\
\hline 52 & Pneumonia & $\operatorname{dotTB}$ \\
\hline 71 & Growth failure & Non TB \\
\hline 111 & Osteomyelitis & $\operatorname{dotTB}$ \\
\hline 128 & Juvenile idiopathic arthritis & $\operatorname{dot} T B$ \\
\hline
\end{tabular}

Footnotes: CMV: cytomegalovirus; TB: tuberculosis; CNS: central nervous system; dotTB: disease other than TB.

doi:10.1371/journal.pone.0138952.t005

immune response in young children. Overall, the number of indeterminate results, which indicate subjects unable to properly respond to the mitogen, was low in the different groups with the only exception being the children enrolled for suspected active TB but diagnosed with a disease different from active TB (dotTB).

Among the 26 children with indeterminate results, bacterial, viral or fungal pneumonia or extra-polmonary infection were later diagnosed in 22 cases $(80.8 \%)$, Table 5 reports the clinical features of all children scoring indeterminate. In Table 3 , the group of children 0-12 months old showed a statistically significant higher rate of indeterminate results $(9 / 52,17.3 \%$; $\mathrm{p}=0.001)$ compared to the other age groups, with the higher proportion among the group classified as dotTB. Conversely, analyses of asymptomatic children screened for LTBI showed that in this group of 481 children, the rate of indeterminate results was particularly low $(0.2 \%)$ and no statistically significant differences were found when the $0-12$ months old group was compared with the older age groups.

To further investigate the possible correlation between inability to respond to mitogen and very young age, in Fig 2 we plotted the IFN- $\gamma$ levels secreted in response to mitogen by children subdivided into 3 main age classes. Only 8 out of 22 (33\%) children $\leq 8$ months old were able to secrete $\geq 10 \mathrm{IU} / \mathrm{ml}$ of IFN- $\gamma$, while $9(41 \%)$ children secreted less than $1.0 \mathrm{IU} / \mathrm{ml}$ of IFN- $\gamma$, 
indicating an impaired ability to respond to mitogen, even though no statistically significant difference was found $(\mathrm{p}=0.23)$, probably due to the low number of evaluated children under 12 months old. Conversely, children older than 8 months responded properly to the mitogen. There was no statistically significant effect of age on the magnitude of the response $(p>0.05)$.

\section{Follow-up}

At follow-up (min. 3 years, max. 6 years), none of the evaluated children who were not initially diagnosed with a disease other than TB (dotTB) received a later diagnosis of active TB.

\section{Discussion}

Diagnosis of TB in children is a challenging task and the reliability of the new IGRAs, which are being effectively used in the adult population, awaits confirmation, primarily in those under 5 years of age. Here, we report the results of a retrospective study carried out on 621 children and we show that young children older than 1 year are capable of properly responding to the mitogenic stimuli and RD1 antigens when infected with $M$. tuberculosis.

In this study, the overall sensitivity of QFT-IT in children with active TB was $87.5 \%$, ranging from $62.5 \%$ in children $25-36$ months old to $100 \%$ in children older than 49 months, in line with previous reports $[5,23]$ and with recent data from a multicenter study in Italy that included only $<2$ years old children, where a sensitivity of $92,4 \%$ for QFT-IT was registered [24]. Conversely, Chiappini E. et al [25] measured a sensitivity of 73,3\% for QFT-IT for active TB in $<5$ years old children, which was much lower than what detected in parallel with TST $(90,0 \%)$. These results highlight the need for more robust results on the use of IGRAs for the diagnosis of active TB, though the data of this study indicate a high sensitivity of QFT-IT. We also observed a high overall (93.6\%) and age-group (>90\%) specificity of QFT-IT for active $\mathrm{TB}$, similarly to what recently observed in other studies [24,25].

The lack of a gold standard for LTBI prevents proper assessment of QFT-IT performance for this group. It is remarkable that 36 out of 38 children (95\%) scoring positive to QFT-IT, and therefore diagnosed with LTBI, had at least one risk factor for TB infection (either known exposure with an adult TB index case, being born in a TB-endemic country or having parents born in a TB-endemic country).

A major concern in the use of IGRA assays in young children is that the potentially immature immune system may result in an impaired capacity to secrete IFN- $\gamma$ in response to antigenic stimuli. The determination of IFN- $\gamma$ in response to mitogen in the QFT-IT assay allows for immune system status assessment and identifying subjects with impaired immunity who result indeterminate. In this study, only 26 children (4.2\%) scored indeterminate with the rate being lowest in children older than 12 months of age (ranging from $6.25 \%$ in children aged $37-48$ months to $1.2 \%$ in children older than 60 months). Conversely, we reported a $17 \%$ of indeterminate results in the 0-12 month-old-children, which is in line with previous data $[15,22,26,27]$. Recently, Critselis et al. found that QFT-IT indeterminate results occurred more frequently among children under 2 years of age (4\%) than children older than 4 (1.7\%) [28]. It has been suggested that subject age may be the main reason for the high level of indeterminate results, although a detailed analysis of the health status of these infants (as provided here) was lacking. In our study, only 2 out of the 26 young children with indeterminate results were asymptomatic children, and one of these two was a 6 -day- old newborn. Two of the children with indeterminate QFT-IT were diagnosed with active TB (one with a severe CNS TB that progressed to exitus) and 11 children were diagnosed with pneumonia.

Among the children who scored indeterminate, Epstein Barr Virus (EBV), Varicella Zoster Virus (VZV) or measles infections were identified in 4 out of the 26 children in our series, 
supporting the findings that ongoing viral infections may be common causes of indeterminate results [22]. Interestingly, 3 children with bacterial lymphadenitis undergoing amoxicillin-clavulanate therapy had indeterminate results, probably because of the ability of $\beta$-lactam benzylpenicillin to bind IFN- $\gamma$ and reduce its availability for detection in the immune assays $[29,30]$. Moreover, 2 children with indeterminate results had juvenile idiopathic arthritis, a well-known cause of a negative TST [31].

Hence, with only one exception, all children with indeterminate scores had a reasonable explanation for the inability to respond to the mitogen, indicating that young age alone (being under 2 years of age, as suggested by the majority of studies) should not be considered the only cause of an indeterminate result, in line with recent findings by Debord et al. [32]. Acute infections seem to be the cause of the high proportion of indeterminate results and for this reason we evaluated the incidence of indeterminate results when an acute infection other than TB was diagnosed.

The performance of the QFT-IT in children living in high burden settings raised some concerns, as highlighted by a study where $27 \%$ of the children tested showed an indeterminate result, with higher rates among the under 2-year-old population and with no statistically significant association with HIV infection, sex or malnutrition [33]. Moreover, an indeterminate QFT-IT result at baseline was associated with subsequent increased childhood mortality [33] in line with previous data [34] and our experience where the only child who died for meningeal TB disease scored indeterminate for the QFT-IT. Although the high rate of indeterminate QFT-IT results in the pediatric population has always been considered an important limitation of this test, the possibility of having a third option in addition to "positive" and "negative" is an important, perhaps underrated advantage of the QFT-IT over the TST. In fact, when there is a high clinical suspicion of TB disease, an indeterminate QFT-IT result could be read as a red flag indicating an important cause of immune suppression or active severe TB requiring urgent and extensive diagnosis $[33,34]$. In this context, the results of this study further highlight the need of a non-adaptive immunity based diagnostic for children in TB endemic countries.

Our study has some potential limitations. First, the TST was performed in less than half of the patients, mainly because of the difficulty the parents had to make a return visit with the children. Hence, we decided not to include TST results in the analyses and to focus on QFT-IT. Second, BCG vaccination was registered in about $40 \%$ of the subjects, mainly due to the lack of valid certification in foreign-born children, thus, we did not include BCG vaccination in the analysis. However, it is well established that BCG vaccination does not impact QFT-IT status. Additional research is needed to improve current immune diagnostic tests for TB, including the use of new M. tuberculosis antigens different from those used in the QFT-IT [35-37], new immunological markers beside IFN- $\gamma$ [38] and/or read-outs different from ELISA [39].

In conclusion, the present study indicates that even young children are able to respond to specific M. tuberculosis antigens and mitogen, providing support for using the QFT-IT assay also in young children, particularly those evaluated for LTBI.

\section{Acknowledgments}

The authors would like to thank Dr Roberta Grossi (UCSC Rome, Italy) for her excellent technical assistance; Andrea Baker for the editing of the manuscript.

\section{Author Contributions}

Conceived and designed the experiments: MiS GD PV DB MaS GF DG. Performed the experiments: MiS GD AZ PD. Analyzed the data: MiS DB GD PV DG. Contributed reagents/materials/analysis tools: MiS GD MaS GF PD AZ. Wrote the paper: MiS GD DB PV DG. 


\section{References}

1. World Health Organization (2012) Global Tuberculosis report 2012.

2. Getahun H, Sculier D, Sismanidis C, Grzemska M, Raviglione M (2012) Prevention, diagnosis, and treatment of tuberculosis in children and mothers: evidence for action for maternal, neonatal, and child health services. J Infect Dis 205 Suppl 2: S216-S227. doi: 10.1093/infdis/jis009 PMID: 22448018

3. Newton SM, Brent AJ, Anderson S, Whittaker E, Kampmann B (2008) Paediatric tuberculosis. Lancet Infect Dis 8: 498-510. S1473-3099(08)70182-8. doi: 10.1016/S1473-3099(08)70182-8 PMID: 18652996

4. Cruz AT, Starke JR (2010) Pediatric tuberculosis. Pediatr Rev 31: 13-25. 31/1/13. doi: 10.1542/pir.311-13 PMID: 20048035

5. Buonsenso D, Lancella L, Delogu G, Krzysztofiak A, Testa A, Ranno O et al. (2012) A 20-Year Retrospective Study of Pediatric Tuberculosis in Two Tertiary Hospitals in Rome. Pediatr Infect Dis J. doi: 10. 1097/INF.0b013e3182615270 PMID: 22668805

6. Ling DI, Pai M, Davids V, Brunet L, Lenders L, Meldau R et al. (2011) Are interferon-gamma release assays useful for diagnosing active tuberculosis in a high-burden setting? Eur Respir J 38: 649-656. doi: 10.1183/09031936.00181610 PMID: 21349910

7. Erkens CG, Kamphorst M, Abubakar I, Bothamley GH, Chemtob D, Haas W et al.(2010) Tuberculosis contact investigation in low prevalence countries: a European consensus. Eur Respir J 36: 925-949. 36/4/925. doi: 10.1183/09031936.00201609 PMID: 20889463

8. Cassone A, Cauda R, De MA (2012) High rate of Quantiferon positive and tuberculin negative tests in infants born at a large Italian university hospital in 2011: a cautionary hypothesis. Pathog Glob Health 106: 8-11. doi: 10.1179/2047773212Y.0000000006 PMID: 22595269

9. Tebruegge M, Salo E, Ritz N, Kampmann B. On Behalf Of The Paediatric Tuberculosis Network European Trialsgroup Ptbnet (2013) Inclusion of latent tuberculosis infection as a separate entity into the international classification of diseases. Thorax 68: 588. thoraxjnl-2012-202824. doi: 10.1136/thoraxjnl2012-202824 PMID: 23128034

10. Barry CE III, Boshoff HI, Dartois V, Dick T, Ehrt S, Flynn J et al. (2009) The spectrum of latent tuberculosis: rethinking the biology and intervention strategies. Nat Rev Microbiol 7: 845-855. doi: 10.1038/ nrmicro2236 PMID: 19855401

11. Menzies RI,. (2000) Tuberculosis skin testing. In: Marcel Dekker, editors. Tuberculosis: A comprehensive International Approach. New York, NY. pp. 279-322.

12. Pai M, Zwerling A, Menzies $D$ (2008) Systematic review: T-cell-based assays for the diagnosis of latent tuberculosis infection: an update. Ann Intern Med 149: 177-184. PMID: 18593687

13. Riazi S, Zeligs B, Yeager H, Peters SM, Benavides GA, Di MO et al. (2012) Rapid diagnosis of Mycobacterium tuberculosis infection in children using interferon-gamma release assays (IGRAs). Allergy Asthma Proc 33: 217-226. doi: 10.2500/aap.2012.33.3574 PMID: 22584190

14. Diel R, Loddenkemper R, Meywald-Walter K, Niemann S, Nienhaus A (2008) Predictive value of a whole blood IFN-gamma assay for the development of active tuberculosis disease after recent infection with Mycobacterium tuberculosis. Am J Respir Crit Care Med 177: 1164-1170. doi: 10.1164/rccm. 200711-16130C PMID: 18276940

15. Diel R, Goletti D, Ferrara G, Bothamley G, Cirillo D, Kampmann B et al. (2011) Interferon-gamma release assays for the diagnosis of latent Mycobacterium tuberculosis infection: a systematic review and meta-analysis. Eur Respir J 37: 88-99. doi: 10.1183/09031936.00115110 PMID: 21030451

16. Dogra S, Narang P, Mendiratta DK, Chaturvedi P, Reingold AL, Colford JM et al. (2007) Comparison of a whole blood interferon-gamma assay with tuberculin skin testing for the detection of tuberculosis infection in hospitalized children in rural India. J Infect 54: 267-276. PMID: 16733068

17. Lighter J, Rigaud M, Eduardo R, Peng CH, Pollack H (2009) Latent tuberculosis diagnosis in children by using the QuantiFERON-TB Gold In-Tube test. Pediatrics 123: 30-37. 123/1/30. doi: 10.1542/peds. 2007-3618 PMID: 19117857

18. Okada K, Mao TE, Mori T, Miura T, Sugiyama T, Yoshiyama T et al. (2008) Performance of an interferon-gamma release assay for diagnosing latent tuberculosis infection in children. Epidemiol Infect 136: 1179-1187. PMID: 17988427

19. National Institute for Health and Clinical Excellence (2006) Tuberculosis: clinical diagnosis and management of tuberculosis, and measures for its prevention and control. In: NICE.

20. Abubakar I, Griffiths $C$, Ormerod $P$ (2012) Diagnosis of active and latent tuberculosis: summary of NICE guidance. BMJ 345: e6828. doi: 10.1136/bmj.e6828 PMID: 23077351

21. Lalvani A, Pareek M (2010) A 100 year update on diagnosis of tuberculosis infection. Br Med Bull 93: 69-84. doi: 10.1093/bmb/ldp039 PMID: 19926636 
22. Haustein T, Ridout DA, Hartley JC, Thaker U, Shingadia D, Klein NJ et al. (2009) The likelihood of an indeterminate test result from a whole-blood interferon-gamma release assay for the diagnosis of Mycobacterium tuberculosis infection in children correlates with age and immune status. Pediatr Infect Dis $\mathrm{J}$ 28: 669-673. doi: 10.1097/INF.0b013e3181a16394 PMID: 19633512

23. Mandalakas AM, Detjen AK, Hesseling AC, Benedetti A, Menzies D (2011) Interferon-gamma release assays and childhood tuberculosis: systematic review and meta-analysis. Int J Tuberc Lung Dis 15: 1018-1032. doi: 10.5588/ijtld.10.0631 PMID: 21669030

24. Garazzino S, Galli L, Chiappini E, Pinon M, Bergamini BM, Cazzato S et al. (2014) Performance of Interferon-y Release Assay for the Diagnosis of Active or Latent Tuberculosis in Children in the First 2 Years of Age. A multicenter Study of the Italian Society of Pediatric Infectious Diseases. Pediatr Infect Dis J 33: e226-e231. doi: 10.1097/INF.0000000000000353 PMID: 25361032

25. Chiappini E, Bonsignori F, Mazzantini R, Sollai S, Venturini E, Mangone G et al. (2014) InterferonGamma Release Assay Sensitivity in Children less than 5 years of Age is Insufficient to Replace the Use of Tuberculin Skin Test in Western Countries. Pediatr Infect Dis J.

26. Connell TG, Ritz N, Paxton GA, Buttery JP, Curtis N, Ranganathan SC (2008) A three-way comparison of tuberculin skin testing, QuantiFERON-TB gold and T-SPOT.TB in children. PLoS ONE 3: e2624. doi: 10.1371/journal.pone.0002624 PMID: 18612425

27. Bergamini BM, Losi M, Vaienti F, D'Amico R, Meccugni B, Meacci M et al. (2009) Performance of commercial blood tests for the diagnosis of latent tuberculosis infection in children and adolescents. Pediatrics 123: e419-e424. 123/3/e419. doi: 10.1542/peds.2008-1722 PMID: 19254978

28. Critselis E, Amanatidou V, Syridou G, Spyridis NP, Mavrikou M, Papadopoulos NG et al. (2012) The Effect of Age on Whole Blood Interferon-Gamma Release Assay Response among Children Investigated for Latent Tuberculosis Infection. J Pediatr. S0022-3476(12)00405-2.

29. Zrinski TR, Zoricic-Letoja I, Pavic I, Dodig S (2011) Indeterminate results of QuantiFERON-TB Gold InTube assay in nonimmunosuppressed children. Arch Med Res 42: 138-143. S0188-4409(11)00014-2. doi: 10.1016/j.arcmed.2011.02.001 PMID: 21565627

30. Brooks BM, Flanagan BF, Thomas AL, Coleman JW (2001) Penicillin conjugates to interferon-gamma and reduces its activity: a novel drug-cytokine interaction. Biochem Biophys Res Commun 288: 11751181. doi: 10.1006/bbrc.2001.5896 S0006-291X(01)95896-6. PMID: 11700035

31. Park JH, Seo GY, Lee JS, Kim TH, Yoo DH (2009) Positive conversion of tuberculin skin test and performance of interferon release assay to detect hidden tuberculosis infection during anti-tumor necrosis factor agent trial. J Rheumatol 36: 2158-2163. jrheum.090150. doi: 10.3899/jrheum.090150 PMID: 19723901

32. Debord C, De LA, Gourgouillon N, Guerin-El K, V, Pedron B, Gaudelus J et al. (2011) Interferongamma release assay performance for diagnosing tuberculosis disease in 0 - to 5-year-old children. Pediatr Infect Dis J 30: 995-997. doi: 10.1097/INF.0b013e3182272227 PMID: 21694659

33. Rose MV, Kimaro G, Nissen TN, Kroidl I, Hoelscher M, Bygbjerg IC et al. (2012) QuantiFERON(R)-TB Gold In-Tube Performance for Diagnosing Active Tuberculosis in Children and Adults in a High Burden Setting. PLoS ONE 7: e37851. doi: 10.1371/journal.pone.0037851 PMID: 22808002

34. Mendez EA, Baquero-Artigao F, Gonzalez-Munoz M, De CF, Mellado Pena MJ, Velazquez-Fragua R (2010) Lack of sensitivity of QuantiFERON-TB gold test in tube in a child with tuberculous meningitis. Pediatr Infect Dis J 29: 683-684. doi: 10.1097/INF.0b013e3181e218b7 PMID: 20574408

35. Delogu G, Chiacchio T, Vanini V, Butera O, Cuzzi G, Bua A et al. (2011) Methylated HBHA produced in M. smegmatis Discriminates between Active and Non-Active Tuberculosis Disease among RD1Responders. PLoS ONE 6: e1815.

36. Goletti D, Carrara S, Vincenti D, Girardi E (2007) T cell responses to commercial mycobacterium tuberculosis-specific antigens in HIV-infected patients. Clin Infect Dis 45: 1652-1654. doi: 10.1086/523012 PMID: 18190334

37. Goletti D, Raja A, hamed Kabeer BS, Rodrigues C, Sodha A, Butera O et al. (2010) IFN-gamma, but not IP-10, MCP-2 or IL-2 response to RD1 selected peptides associates to active tuberculosis. J Infect 61: 133-143. doi: 10.1016/j.jinf.2010.05.002 PMID: 20470822

38. Kabeer BS, Raja A, Raman B, Thangaraj S, Leportier M, Ippolito G et al. (2011) IP-10 response to RD1 antigens might be a useful biomarker for monitoring tuberculosis therapy. BMC Infect Dis 11: 135. 1471-2334-11-135. doi: 10.1186/1471-2334-11-135 PMID: 21595874

39. Rozot V, Vigano S, Mazza-Stalder J, Idrizi E, Day CL, Perreau M et al. (2013) Mycobacterium tuberculosis-specific CD8+ T cells are functionally and phenotypically different between latent infection and active disease. Eur J Immunol 43: 1568-1577. doi: 10.1002/eji.201243262 PMID: 23456989 